\title{
Attosecond electron bunch measurement with coherent nonlinear Thomson scattering
}

\author{
Colton Fruhling $\odot,{ }^{*}$ Grigory Golovin, and Donald Umstadter® \\ Department of Physics and Astronomy, University of Nebraska-Lincoln, \\ Behlen Laboratory 500 Stadium Drive, Nebraska 68588, USA
}

(Received 3 March 2020; accepted 5 June 2020; published 6 July 2020)

\begin{abstract}
We present a novel method for measurement of ultrashort electron-bunch duration, in principle, as short as zeptosecond $\left(10^{-21} \mathrm{~s}\right)$. The method employs nonlinear Thomson scattering of relativistically intense laser light, and takes advantage of the nonlinear dependence and coherence of scattered light on electron bunch length. We validate the method and test its range of applicability via simulations by using realistic (nonideal) electron beams. Due to the wide flexibility in choice of interaction geometry and scattering laser pulse properties enabled by the method, it is shown to be applicable over a wide range of electron beam parameters, including energy, energy spread, and divergence angle.
\end{abstract}

DOI: 10.1103/PhysRevAccelBeams.23.072802

\section{INTRODUCTION}

With the advent of ultrashort duration lasers and electron accelerators, the study of ultrafast phenomena has rapidly advanced. Dynamics on the attosecond timescale can now be probed, opening a door to previously unexplored physics realms [1]. High-charge attosecond-duration electron bunches are at the frontier of accelerator-driven free-electron lasers, inverse Compton scattering light sources, and ultrafast electron diffraction sources. A number of concepts for attosecond electron-bunch generation have been theoretically proposed, including laser-illuminated droplets [2], direct acceleration from focusing to a $\lambda^{3}$ volume [3], laser wakefield acceleration (LWFA) with localized injection on sharp density up-ramps [4], ponderomotive bunching from optical pulses [2], temporal lensing of electron bunches [5], dielectric laser acceleration [6] and optically seeded free-electron lasers [7].

Current techniques for characterizing the ultrashort durations of electron bunches include time-space methods, such as streaking via laser ponderomotive scattering [8], direct laser field streaking [9], and cross-correlation [10]. Another way to measure electron-bunch duration utilizes changes in the frequency space and coherence of broadband radiation emitted by the bunch. Electron bunches radiate coherently for wavelengths greater than the bunch size, and incoherently for wavelengths smaller than the bunch size. The transition from coherent to incoherent regimes can be

*colton.fruhling@huskers.unl.edu

Published by the American Physical Society under the terms of the Creative Commons Attribution 4.0 International license. Further distribution of this work must maintain attribution to the author(s) and the published article's title, journal citation, and DOI. observed in the spectrum of the emitted radiation and used to measure the bunch length. This has been demonstrated experimentally with early synchrotrons [11].

Two such methods are Smith-Purcell radiation (SPR) $[12,13]$ and coherent transition radiation (CTR) [14], which has measured a single 1.5-fs electron bunch. Another method of current interest is optical diffraction radiation [15]. All of these methods require that the radiation emitted by the electron bunch is broadband and covers a wavelength range on the order of the electron bunch length. However, the intensity of the radiation produced by CTR and SPR typically decays above frequencies necessary to measure attosecond electron bunches. For CTR, radiation decays rapidly, as $\sim\left(\gamma \omega_{p} / \omega\right)^{4}$, above the relativistically shifted plasma frequency, $\omega=\gamma \omega_{p}$ [16] of the transition material; for SPR, it decays as $\sim e^{-2 \omega d / \gamma \beta c}$ above a critical frequency $\omega=\gamma \beta c / 2 d[17,18]$, where $\gamma$ and $\beta$ are the relativistic energy and velocity respectively, $d$ is the distance the electron passes above the Smith-Purcell grating surface, and $c$ is the speed of light in vacuum.

The areas of applicability of these methods for typical experimental parameters are shown in Fig. 1 as shaded regions in order for the reader to orient oneself with the problem space. As one can see, both CTR and SPR for typical experimental conditions (see caption for details) cannot be used to measure attosecond electron bunches at energies near $\gamma \leq 10$, which is one of the primary regions of interest for attosecond physics [19-21].

In this paper, we introduce a novel solution to the problem of measuring ultrashort electron bunch duration, using the mechanism of nonlinear Thomson scattering (NTS). It has previously been shown that linear Thomson scattering could be used to measure transverse emittance and energy of electron beams [24-26], however NTS has never been employed before, and never for bunch-duration measurements. In this 


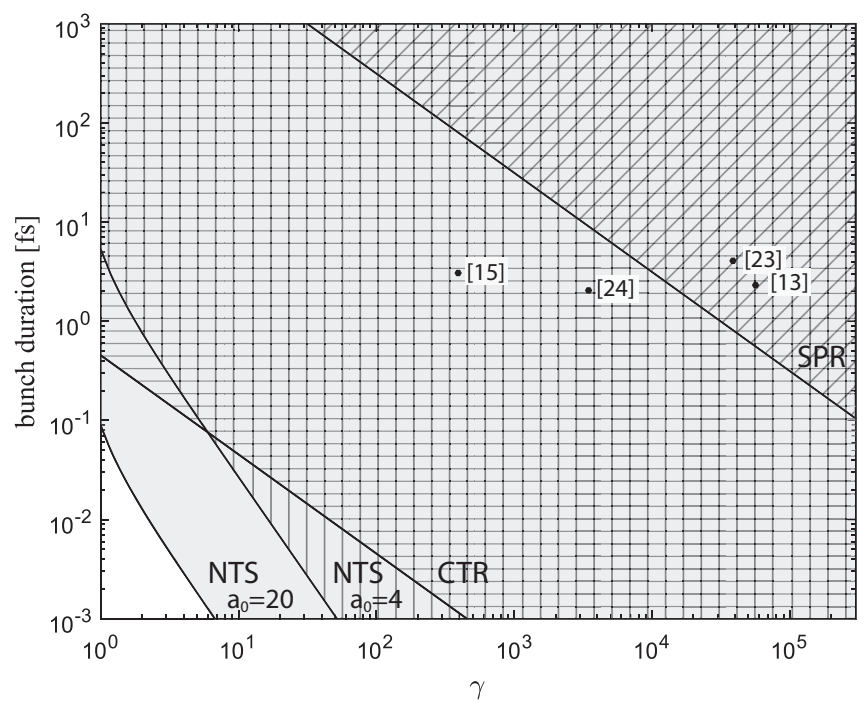

FIG. 1. The minimum bunch duration measurement possible based on ideal detection for SPR (diagonal hatched), CTR (horizontally hatched), and NTS for $a_{0}=4$ (vertically hatched) and $a_{0}=20$ (shaded) under typical experimental conditions. The dimensionless laser intensity parameter, $a_{0}$, is defined later in the text. The shading indicates applicability that extends to the top right. SPR [12,22] is considered in the case where the distance of the beam from the grating is $d=1 \mathrm{~mm}$. CTR [14,23] is considered in the case where aluminum, a common material, is used for a transition material. NTS is considered with the parameters used in this paper, $a_{0}=4$, and at state-of-the-art systems where $a_{0}=20$.

method, NTS overcomes the limitations of SPR and CTR by generating broadband radiation at higher frequencies, and yet with lower electron energies. NTS occurs when an electron oscillates in an intense laser field and exhibits a complicated relativistic trajectory, which emits broadband radiation that can extend far into the sub-nm spectrum. Modern laser systems can easily reach the intensity regimes required $\left(a_{0}=1-20\right)[27,28]$. Figure 1 shows that NTS by a mildly relativistic $(\gamma \sim 1-1000)$ electron bunch can be used to measure electron pulse durations as low as attosecond, and potentially even zeptosecond.

The paper is organized as follows. Section II presents a short review of coherent radiation and the theory required to analyze electron bunch duration via coherency effects. Next, we present NTS and aspects of the radiation such as the cutoff energies and tunability parameters. Section III describes numerical simulations used to model the proposed method. A simple analysis is used to demonstrate the validity of the method. Finally, we discuss limitations of the method due to nonideal electron beam conditions.

\section{REVIEW}

\section{A. Coherent radiation}

We start by considering a group of radiators, such as electrons, where the instantaneous total radiation of the group can be expressed as
$I_{\mathrm{tot}}=I_{\mathrm{inc}}(\omega)\left|\sum_{j}^{N} e^{i \frac{\omega}{\tilde{c}} \hat{n} \cdot \vec{r}_{j}}\right|^{2}=I_{\mathrm{inc}}(\omega)\left(N+\sum_{j \neq k}^{N} e^{i \frac{\omega}{c} \hat{n} \cdot\left(\vec{r}_{j}-\vec{r}_{k}\right)}\right)$,

where the summation contains all electrons in the bunch, $\hat{n}$ is the direction of observation, $\vec{r}_{j}$ and $\vec{r}_{k}$ are electron positions, and $I_{\mathrm{inc}}(\omega)$ is the intensity of radiation of a single electron. The first term is simply the incoherent addition of each single electron and is proportional to $N$, whereas the second term contains information about the distribution of the bunch. One can immediately see that the radiation for a particular wavelength, $\lambda$, will be considered coherent if the phase terms are all identical. If each electron bunch length is $\delta$, then coherency occurs either when $\delta / \lambda \ll 1$ or $\omega \tau \ll 2 \pi$, where $\tau=\delta / c$. Equivalently, the grouping of electrons is so small compared to the wavelength of radiation emitted that they can emit in phase with each other. If this happens for many wavelengths within the broadband spectrum, we will be able to recover $\delta$.

The second term in Eq. (1.1) can be represented in a more useful form by averaging over the particle positions and assuming they are uncorrelated. [The correlation function is $C\left(\vec{r}, \vec{r}^{\prime}\right)=S(\vec{r}) S\left(\vec{r}^{\prime}\right)$, where $S(r)$ is the normalized density function of the group of electrons.] Finally, as was shown in [29], Eq. (1.1) can be written as

$$
I_{\text {tot }}(\omega)=I_{\text {inc }}(\omega)[N+N(N-1) F(\omega)],
$$

where $F(\omega)$ is the form factor

$$
F(\omega)=\left|\int d r^{3} S(\vec{r}) e^{i \frac{\omega}{c} \hat{n} \cdot \vec{r}}\right|^{2}=|\hat{S}(\omega, \hat{n})|^{2} .
$$

In order to measure electron bunch duration via coherent radiation, one measures the spectral intensity $I_{\text {tot }}(\omega)$ of emitted radiation in a particular direction, divides by the theoretical or experimental incoherent signal to find $F(\omega)$, and then performs an inverse Fourier transform to recover the projection of $S(\vec{r})$ along the observation direction.

Of course, the phase retrieval problem [30] results from experimentally measuring the magnitude $F(\omega)$, but not the density function $\hat{S}(\omega, \hat{n})$, which in general requires the phase. Although this issue is outside the scope of this paper, the authors direct an interested reader to Refs. [30-33]. To simplify the analysis, we employ a constant-phase approximation, setting the phase for each wavelength to be constant. This allows us to ignore the phase and more simply take the Fourier transform of the magnitude of $F(\omega)$.

\section{B. Nonlinear Thomson scattering}

Thomson scattering occurs when light is scattered by a free electron. In linear scattering, an electron in a weak laser field oscillates along the electric field and radiates at the 
laser frequency $[34,35]$. If the electron is moving relativistically, the radiation is emitted in a narrow cone, $\theta_{\text {rad }} \sim 1 / \gamma$, pointed in the direction of average electron motion, and the emitted frequency is double-Doppler shifted from the laser frequency [see Eq. (1.4)]. This case is often also referred to as inverse Compton scattering. As the laser intensity increases, the magnetic component of the Lorentz force then becomes comparable to the electric force, and the equation of motion becomes nonlinear in the fields. The dimensionless laser parameter $a_{0}=e E / m_{e} c \omega=$ $0.85 \times 10^{-9} \lambda_{0}[\mu \mathrm{m}] I^{1 / 2}\left[\mathrm{~W} / \mathrm{cm}^{2}\right]$ describes the onset of nonlinear behavior in the system.

In the highly nonlinear regime, $a_{0} \gg 1$, which is the regime required for our measurement, and the radiation becomes broadband, resulting in a synchrotron-like spectrum as demonstrated experimentally by $[27,28]$. For the application of electron bunch measurement, we are most interested in the minimum and maximum frequency achievable from NTS, which defines our limit of applicability. The minimum frequency is easily understood as the Doppler-shifted laser frequency with an angular and ponderomotive modification and has been shown to be described by

$$
\omega_{\text {fund }}=\frac{2 \gamma^{2}(1-\boldsymbol{\beta} \cdot \hat{\mathbf{k}}) \omega_{0}}{1+\gamma^{2} \theta^{2}+a_{0}^{2} / 2}
$$

where $\boldsymbol{\beta}$ and $\gamma$ are the initial electron velocity and energy respectively, $\omega_{0}$ and $\hat{\mathbf{k}}$ are the laser frequency and direction, and $\theta$ is the angle between the average direction of electron motion and the direction of observation. The maximum frequency is slightly harder to define due to the broadband nature of the radiation, although the precedent has been that it is the frequency above which the radiation is insignificant for all angles, and is defined [35] as

$$
\omega_{\text {crit }} \approx \frac{3 a_{0}^{3}}{4} \frac{\gamma^{2}(1+\beta)^{2}}{\left(1+\frac{a_{0}^{2}}{2}\right)} \omega_{0} .
$$

With the required background now complete, we now address the full picture of the proposed method. The results of Eqs. (1.4) and (1.5) allows us to understand the unique versatility of NTS for coherent bunch measurement. In the synchrotron-like spectrum, the lowest frequency emitted is the fundamental, which can be tuned by adjusting the laser intensity or the interaction geometry. However, we also desire control of the highest frequency. Thus, we can have control of the minimum frequency via the interaction angle and the highest frequency via the nonlinearity of the interaction.

Consider a bunch of relativistically moving electrons $(\gamma \approx 10)$ that have a duration of 10 attoseconds. The electron bunch is irradiated by an intense $\left(a_{0}=4\right)$ laser pulse $(\lambda=800 \mathrm{~nm})$, and the radiation emitted from this

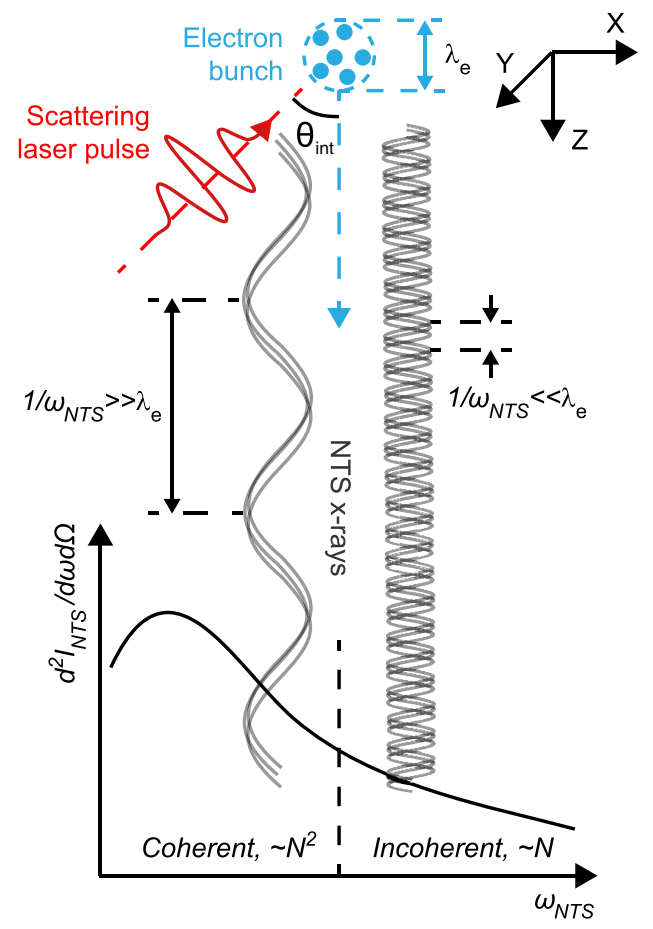

FIG. 2. A schematic of the electron pulse measurement concept. An intense laser pulse (red) interacts with a relativistically moving electron bunch (blue). The interaction angle $\cos \theta_{\text {int }}=$ $\left\langle\vec{\beta}_{0}\right\rangle \cdot \hat{k}$ is defined by the angle between the incoming laser pulse and the average electron bunch direction of motion, which is defined to be along the positive $\mathrm{z}$ axis. The radiation emitted is shown below the interaction and is composed of many different frequencies, which are coherent or incoherent based on whether the relation $\omega \lambda_{e} / 2 \pi c$ is less than or greater than 1 . From the spectrum, which displays the change from coherent to incoherent, one can reconstruct the bunch length.

interaction is measured at some distant detector. These parameters of the laser were chosen so that using Eq. (1.4) we calculate $\omega_{\text {fund }} \tau_{\text {bunch }} \approx 1 \ll 2 \pi$ and using Eq. (1.5) we calculate $\omega_{\text {crit }} \tau_{\text {bunch }}=200 \gg 2 \pi$. This ensures that the radiation consists both of wavelengths that are longer than the bunch length and are emitted coherently as shown on the left side of Fig. 2, and wavelengths that are shorter than the bunch and are emitted incoherently, like that shown on the right side of Fig. 2. Then, the measured spectrum can be Fourier transformed to reconstruct the electron bunch duration.

\section{RESULTS}

This section describes the validation of the proposed method through electromagnetic simulations of electrons interacting with an intense laser field. The simulation procedure is similar to other well-established methods already published in Refs. [36,37]. First, an electron trajectory is calculated in a laser field. The laser field was modeled under the standard paraxial approximation, 
which is valid for loose focusing considered here. Next, the trajectory was used in concert with the Liénard-Wiechert potentials to calculate the retarded electric fields at the detection point, which is in the far field compared to the wavelength of all radiation emitted. Each electron is time stamped such that the radiation emitted from it can be assigned to a global time to include coherency effects. The far-field approximation is only used in the calculation of the electric field amplitude; however, there are no approximations made when calculating the retarded phase. The geometry of the interaction can be understood from Fig. 2.

\section{A. Ideal electron beam}

We first run an initial trial case with ideal electron parameters to test the reconstruction method. The ideal electron beam had no transverse size in the $x-y$ plane with a normal distribution of electrons along the $z$ axis with a 9nm FWHM ( $\sim 3$ as) — see the black line in Fig. 3(c). All electrons had an energy of $\gamma=10$ with the momentum solely in the positive $\mathrm{z}$ direction. For this case and all cases below, the laser was taken to be traveling along the $\mathrm{z}$ axis in the $-z$ direction $\left(\theta_{\text {int }}=\pi\right)$. The laser had the following parameters: wavelength $\lambda_{\text {laser }}=800 \mathrm{~nm}$, relativistic laser potential $a_{0}=4$, spot size $25 \mu \mathrm{m} \sim 30 \lambda_{\text {laser }}$, which validates the use of the paraxial approximation. The laser is brought to focus at the origin where electrons' phase space is defined.

Figure 3(a) shows the on-axis NTS radiation spectrum for a single electron (dark blue line) and for an ideal 5000electron bunch (red line). One notices the general features of the single electron spectrum discussed in Sec. II B in that it is synchrotron-like with a tail visible at high frequencies, and has a low frequency cutoff at $\sim 44 \omega_{\text {laser }}$ which can be understood as the fundamental frequency of NTS predicted by Eq. (1.4). Comparing the electron bunch spectrum to the single electron case, we can see evidence of coherency in the lower frequencies-above the fundamental-that extends up to $\sim 128 \omega_{\text {laser }}$. The radiation amplitude is $10^{6} \sim$ $N^{2}$ of the single-electron case, which points to coherent addition of emissions of single electrons within the bunch. Beyond the high-frequency cutoff, however, the spectrum begins to resemble the single-electron one, and the amplitude drops to $10^{3} \sim N$, meaning an incoherent addition of emissions of single electrons. The loss of coherency occurs
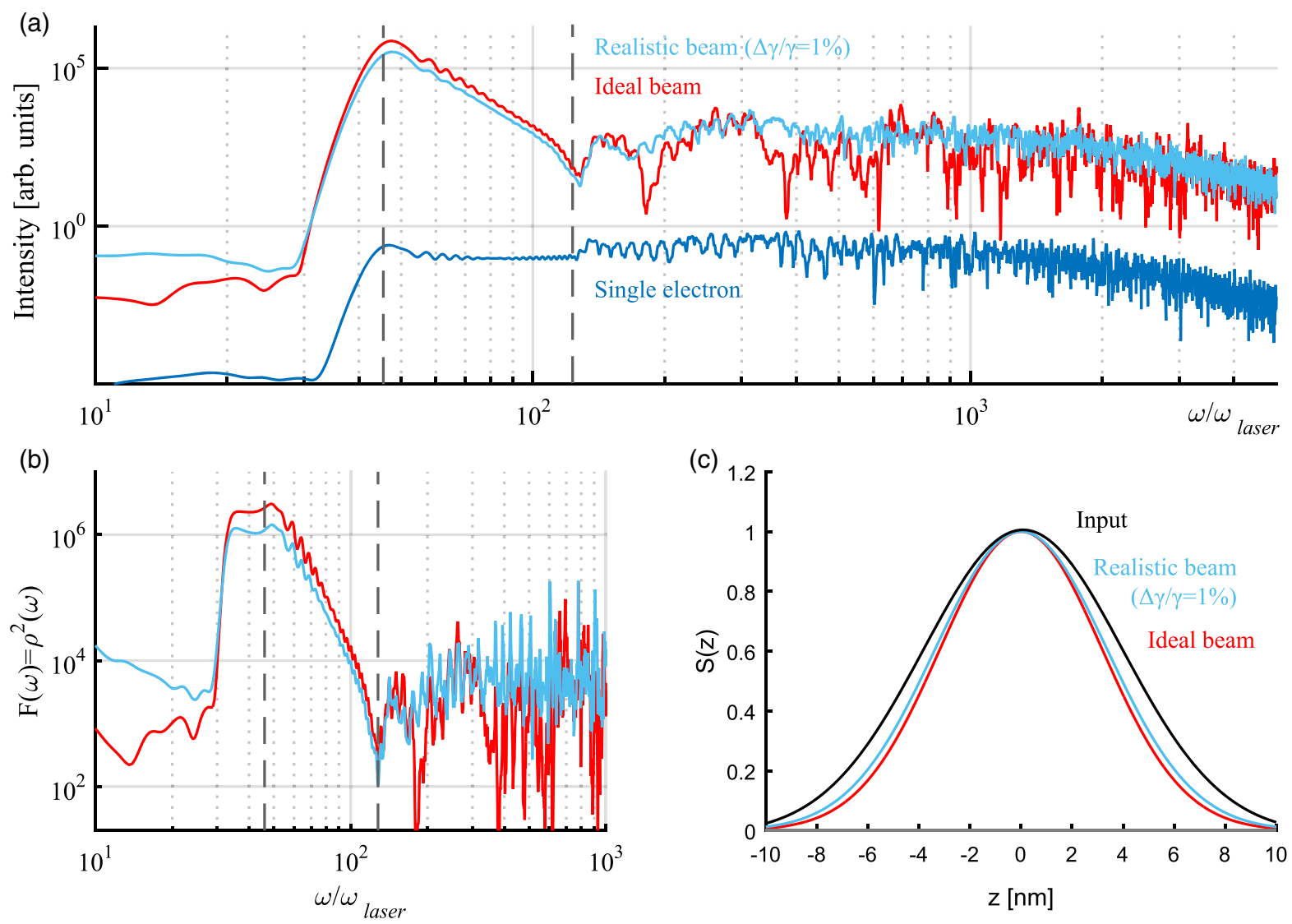

FIG. 3. Simulation results for the ideal and realistic beam conditions. The laser intensity is $a_{0}=4$, spot size $25 \mu \mathrm{m}$. The electron energy is $\gamma=10$. (a) The NTS radiation spectra for a single electron, ideal, and realistic electron beams. (b) Form factors $F(\omega)$. The analysis region is shown between the vertical dashed lines. (b) The reconstructed bunch profiles and their comparison to the simulation input. 
near $100 \omega_{\text {laser }}$ or $\lambda_{\text {laser }} / 100=8 \mathrm{~nm}$, which is consistent with the picture of coherency developed earlier considering the initial bunch length of $9 \mathrm{~nm}$.

The ideal case exhibits clear drops in signal at $\sim 200 \omega_{\text {laser }}$ and $\sim 400 \omega_{\text {laser }}$. These are present for two reasons. First, because the particle distribution is discrete, there must be a hard cutoff where the distribution stops abruptly. Thus the distribution is more of a Gaussian imposed upon a square pulse, and the Fourier transform performed in Eq. (1.3) will resemble a sinc function. The dips correspond to the zeros of the square of the sinc function. Second, every electron in this ideal distribution has the same initial momentum and impinges upon the laser pulse in exactly the same manner, except for the slight spatial separation. Each electron then emits exactly the same radiation, except at slightly different times, and this coherency is unrealistically amplified. The realistic beam presented in Fig. 3(a) exhibits a smoother spectrum because the small changes in position and energy cancel each other.

To limit the noise in our subsequent reconstruction analysis, we chose the low- and high-frequency cutoffs at $44 \omega_{\text {laser }}$ and $128 \omega_{\text {laser }}$ respectively [the dashed lines in Fig. 3(b)]. The coherency is more evident in Fig. 3(b), where $F(\omega)$, the form factor, is plotted and also shows a clear coherency between the two dashed lines where the maximum amplitude is approximately $10^{6} \sim N^{2}$. If we assume the electron bunch is Gaussian in nature, then a Fourier transform of the density function, $S(\vec{r})$, will again produce a Gaussian in the form factor, $F(\omega)$. Therefore, we fit the coherent part of the spectrum, within our limits, to a Gaussian, and the width of the density function is the inverse of the width of the fit. The result is shown in Fig. 3(c) and is compared to the input density function. The results are in good agreement with the input and validate the reconstruction method.

Figure 4 demonstrates the effect of bunch length on the spectrum, using the same initial conditions for the ideal beam considered in Fig. 3. The spectrum for a 4.5, 9, and $18 \mathrm{~nm}$ bunch length are shown. The low end of the spectrum exhibits the fundamental cutoff, which is not affected by beam size. We note the expected degree of coherency decreases with the increase in beam size. Additionally, the first minimum in the spectrum shifts in frequency as expected considering beam size. For a beam that is twice shorter $(4.5 \mathrm{~nm})$, the minimum shown as a dot-dashed line shifts by an amount that is double that of the $9 \mathrm{~nm}$ beam, which is shown as a dotted line. Also, for twice longer bunch length $(18 \mathrm{~nm})$, the minimum, shown as a dashed line, shifts in frequency by an amount that is half that of the $9 \mathrm{~nm}$ beam. This trend is also consistent with the coherency discussed earlier, and provides a reliable estimate of beam size.

\section{B. Realistic electron beams}

From the fundamental point of view, our method should be applicable if the spectrum of each electron sufficiently overlaps all others, such that coherent addition can take

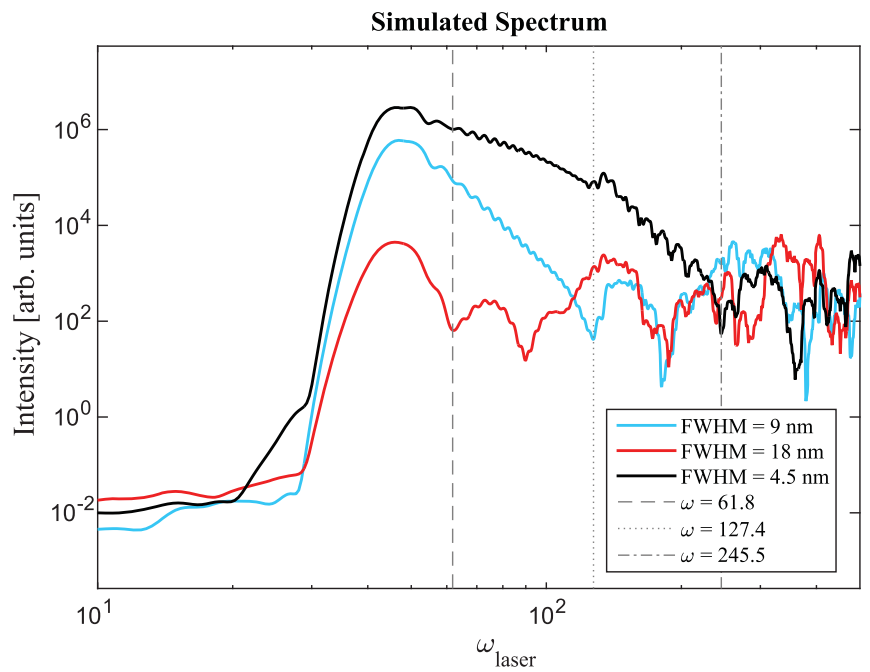

FIG. 4. Simulation results for the ideal beam conditions. The laser intensity is $a_{0}=4$, spot size $25 \mu \mathrm{m}$. The electron energy is $\gamma=10$. The beam size is $4.5,9$, and $18 \mathrm{~nm}$ respectively. The spectrum is shown for each case as well as a dashed, dotted, and dot-dashed vertical line at first minimum of the spectrum for each case respectively.

place. In this section we will test this hypothesis, and discuss the feasibility of the method in the case of realistic laboratory conditions. We focus on attosecond electron bunches generated during laser-plasma interactions, of which there are proposed methods discussed in the Introduction. In the previous simulation, we neglected energy spread, divergence angle, and transverse size of electron beams; we now consider their effects. We keep the assumption that the position and momentum parameters used to describe the electron distribution vary smoothly and have a single peak. This is a valid assumption for most accelerators, and implies the variation of parameters is then mapped to the radiated spectrum in a smooth manner. Any discontinuity or multiple peaks may lead to errors or ambiguities in reconstruction.

We begin by investigating electron-beam size and divergence, since they manifest themselves in a similar way. For a finite beam size and an on-axis NTS measurement, the radiation from an electron on the outside of the bunch will be seen by the detector at a certain angle to the electron bunch propagation direction, resulting in a downshift of the fundamental frequency, as well as the rest of the spectrum. Similarly, for an electron not traveling exactly towards the detector, the radiation from that electron will be directed in its respective propagation direction and so the detector will see the off-axis contributions from that electron. Both effects result in nonzero $\theta$ in Eq. (1.4). The effects can be neglected as long as the radiation cone $\theta_{\mathrm{rad}} \sim 1 / \gamma$ is much larger than the solid angle of the electron bunch, as seen by the detector, and the bunch divergence. Additionally, due to the angular dependence of the emission spectrum, the solid angle of acceptance of the spectrometer used with this diagnostic method should be 
much smaller than the radiation cone of the scattered light. Because of the small size of the Thomson cross section, a low number of detectable scattered photons are expected. Thus, one viable spectroscopy technique is based on x-ray detection with a single-photon-counting array, which has been experimentally demonstrated [26,38].

The effect of energy spread is much harder to consider due to the complex dependence of the emitted NTS spectrum on $\gamma$. Consider first an electron whose energy is less than average energy. The spectral overlap between that electron and the bunch deteriorates because the critical frequency position of the spectrum decreases with $\gamma^{2}$, and the intensity of the radiation above that decays rapidly. For an electron with energy above average, the overlap deteriorates due to the fundamental cutoff [Eq. (1.4)]. Additionally, the intensity of the spectrum scales by $\sim \gamma^{2}$ so initially there may be an increase of overlap, but the fundamental cutoff will eventually take effect. The intensity scaling also means that the measurement is skewed to higher energy electrons because they will contribute more to the total intensity. If there were some spatial-energy chirp to the electron bunch, the method would underestimate the bunch length because of the heavier weighted high-energy electrons.

Finally, if the duration of the interaction between the laser and electrons is such that the bunch length changes considerably, the incoherent radiation could dominate, and a measurement may not be possible. We demonstrate here that the NTS technique can still accurately diagnose electron bunch length for dispersive electron beams. For example, even though the electron bunch in our simulation temporally stretches by 30 attoseconds, knowledge of its energy $(\gamma=10)$ and energy spread (1\%) allowed us to nonetheless determine its bunch length. With the great complexity to consider, it is best to simulate the interaction for energy spreads greater than $1 \%$ and decide if the method is applicable.

Another consideration for experimental realization of this diagnostic technique is the quality and characteristics of the laser light. For example, the pointing instability of the laser beam may affect its overlap with the electron bunch, causing the effective laser intensity experienced by the electrons during the interaction to be smaller than expected. Consequently, the emitted NTS spectrum will not contain the necessary frequencies for electron bunch length measurement. This problem can be mitigated by determining an effective laser intensity from the high energy cutoff of the NTS spectrum, and using it [Eq. (1.5)] and adjusting laser parameters accordingly.

We tested two sets of parameters, one typical for proposals on generation of attosecond-long LWFA beams and another in a regime closer to attosecond-long electron beams created via energy chirp compression. In both sets the beams had a transverse size of $1 \mu \mathrm{m}$ and divergence angle of $5 \mathrm{mrad}$, the electron beams' energy was centered around $\gamma=10$ with a $1 \%$ and $40 \%$ energy spread; all other parameters of the simulation were the same as above. The results for 1\% energy spread are shown in Fig. 3 in light blue. Since the spot size and divergence are below the method's criteria of applicability discussed earlier, NTS spectral corrections are small. Thus, a spectrum of a single electron with an average beam energy can be used for reconstruction. Figure 3(c) shows that our method gives an
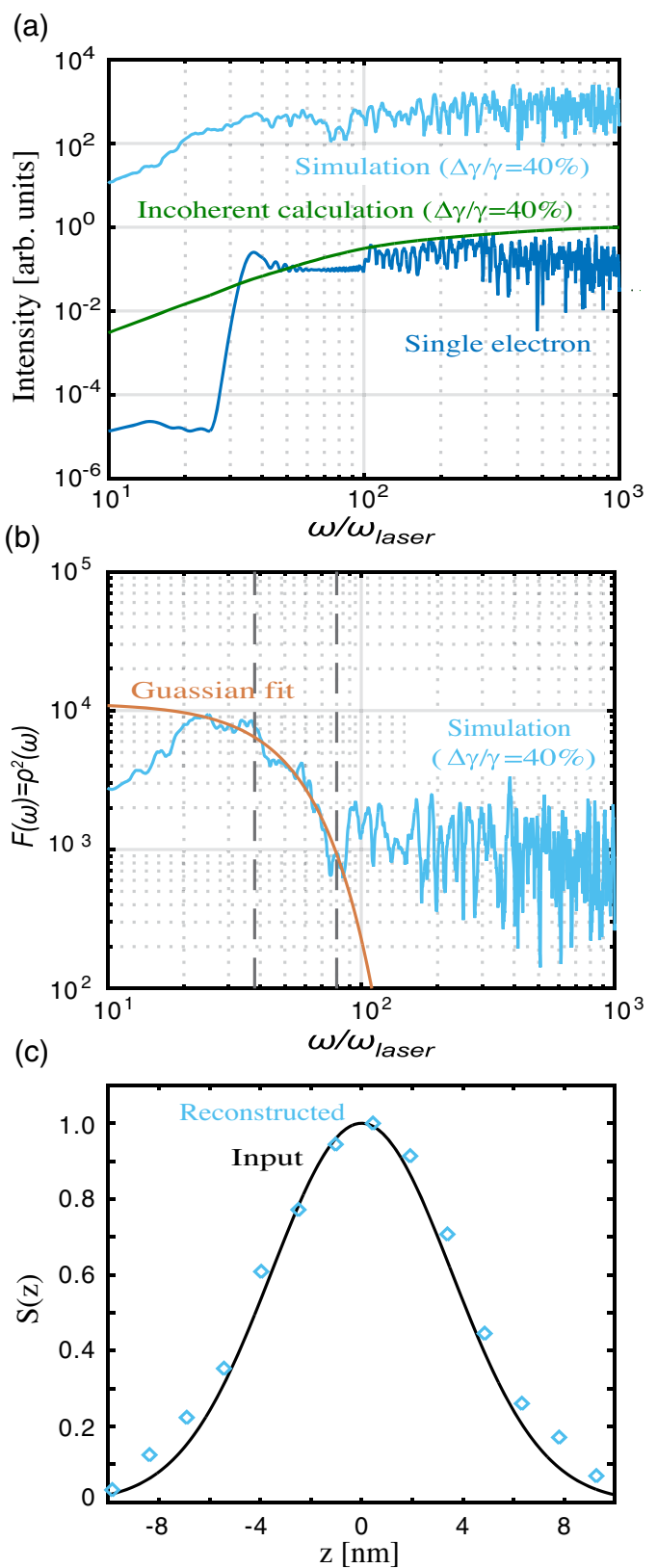

FIG. 5. The radiation for a single electron and a bunch of 5000 are plotted on the left. As can be seen the single electron spectrum is a poor representation for the many electron case and a calculation using knowledge of the input electron energy spectrum is used instead and shown in green. The green curve is used as an incoherent signal to find the form factor, shown in the middle in log-log scale. The Gaussian fit is shown in red and the bounds of fitting are dashed vertical lines. The reconstruction, on the right, recovers the bunch length with good fidelity. 
estimation of the electron bunch duration at 2.67 as $(8 \mathrm{~nm})$, which matches the input value of 3 as $(9 \mathrm{~nm})$ well and almost perfectly matches the ideal case.

The case with $40 \%$ energy spread, however, requires a more informed analysis. Figure 5(a) shows the spectrum for a single electron (dark blue) and for the $40 \%$ energy spread case (light blue). The spectra do not match well because in the realistic case there are electrons with energies much lower than $\gamma=10$ and thus the low NTS frequency cutoff is not as sharp as in a single-electron case. However, instead of using a single-electron spectrum, one can use the energy spectrum of the electron beam, which can be easily measured independently, and calculate the corresponding incoherent NTS spectrum based on it [see the green curve on Fig. 5(a)]. The analysis proceeds as before by dividing the measured NTS signal by the incoherent spectrum and fitting the selected area of the spectrum to a Gaussian shape shown in Fig. 5(b). Figure 5(c) shows that when done in this way, the reconstruction is a perfect match (it gives 3 as with the input value of 3 as) even for electron beams with large and realistic energy spreads.

\section{CONCLUSION}

We explored a novel method for measuring the duration of relativistic attosecond electron bunches. NTS is demonstrated to be a viable technique for generating broadband radiation in a frequency range suitable for analysis of bunch length via coherent effects. The radiation emitted from an ultrashort bunch of electrons was simulated and then standard techniques were employed to correctly deduce the electron bunch duration. Limits on characteristics of the electron beam were discussed based on physical arguments. Finally, realistic electron beam parameters were tested, and reconstructions were shown to be in good agreement with inputs, validating the method.

It is possible to expand upon the reconstruction method used here and employ the more sophisticated ones mentioned earlier. It is also possible to expand from one- to three-dimensional reconstruction without significant changes to the setup, since the form factor at a particular observation angle is the projection of the bunch density distribution along that direction, and one could collect spectra at many different angles and reconstruct a 3D image of the electron bunch. The details of this procedure are discussed by Lai and Sievers [29].

Finally, although attosecond electron beams generated from the schemes mentioned in the introduction have yet to be realized, this method is also applicable to longer-duration laser wakefield electron beams. Our NTS technique could have been used to measure the recently reported 1.5 -fs electron pulse [23] generated with LWFA. If one uses a laser with wavelength of $1 \mu \mathrm{m}, a_{0}=10$, and an interaction angle of $135^{\circ}$, the fundamental frequency of NTS would be shifted to the optical region and $\omega_{\text {fund }} \tau_{\text {bunch }}=3.2<2 \pi$, such that coherent radiation could be measured and used for pulse duration measurements, as described above. Thus, we believe our method has the potential to substantially advance an important frontier of ultrafast dynamics.

\section{ACKNOWLEDGMENTS}

This material is based upon work supported by the U.S. Department of Energy, High-Energy Physics, under Award No. DE-SC0019421 (Controlled Injection of Electrons for the Improved Performance of Laser-Wakefield Acceleration), and the U.S. Department of Energy, Office of Science, Basic Energy Sciences, under Award No. DE-FG0205ER15663 (Laser-Driven X-rays for Ultrafast Science).

[1] F. Krausz and M. Ivanov, Attosecond physics, Rev. Mod. Phys. 81, 163 (2009).

[2] T. V. Liseykina, S. Pirner, and D. Bauer, Relativistic Attosecond Electron Bunches from Laser-Illuminated Droplets, Phys. Rev. Lett. 104, 095002 (2010).

[3] N. M. Naumova, J. A. Nees, I. V. Sokolov, B. Hou, and G. A. Mourou, Relativistic Generation of Isolated Attosecond Pulses in a $\lambda^{3}$ Focal Volume, Phys. Rev. Lett. 92, 063902 (2004).

[4] F. Y. Li, Z. M. Sheng, Y. Liu, J. Meyer-Ter-Vehn, W. B. Mori, W. Lu, and J. Zhang, Dense Attosecond Electron Sheets from Laser Wakefields using an Up-Ramp Density Transition, Phys. Rev. Lett. 110, 135002 (2013).

[5] S. A. Hilbert, C. Uiterwaal, B. Barwick, H. Batelaan, and A. H. Zewail, Temporal lenses for attosecond and femtosecond electron pulses, Proc. Natl. Acad. Sci. U.S.A. 106, 10558 (2009).

[6] M. Kozák, J. McNeur, K. J. Leedle, H. Deng, N. Schönenberger, A. Ruehl, I. Hartl, J. S. Harris, R. L. Byer, and P. Hommelhoff, Optical gating and streaking of free electrons with sub-optical cycle precision, Nat. Commun. 8, 14342 (2017).

[7] C. M. S. Sears, E. Colby, R. Ischebeck, C. McGuinness, J. Nelson, R. Noble, R. H. Siemann, J. Spencer, D. Walz, T. Plettner, and R. L. Byer, Production and characterization of attosecond electron bunch trains, Phys. Rev. ST Accel. Beams 11, 061301 (2008).

[8] C. T. Hebeisen, R. Ernstorfer, M. Harb, T. Dartigalongue, R. E. Jordan, and R.J. D. Miller, Femtosecond electron pulse characterization using laser ponderomotive scattering, Opt. Lett. 31, 3517 (2006).

[9] F. O. Kirchner, A. Gliserin, F. Krausz, and P. Baum, Laser streaking of free electrons at $25 \mathrm{keV}$, Nat. Photonics 8, 52 (2014).

[10] W. P. Leemans, R. W. Schoenlein, P. Volfbeyn, A. H. Chin, T. E. Glover, P. Balling, M. Zolotorev, K. J. Kim, S. Chattopadhyay, and C. V. Shank, X-Ray Based Subpicosecond Electron Bunch Characterization using $90^{\circ}$ Thomson Scattering, Phys. Rev. Lett. 77, 4182 (1996).

[11] T. Nakazato, M. Oyamada, N. Niimura, S. Urasawa, O. Konno, A. Kagaya, R. Kato, T. Kamiyama, Y. Torizuka, T. Nanba, Y. Kondo, Y. Shibata, K. Ishi, T. Ohsaka, and M. Ikezawa, Observation of Coherent Synchrotron Radiation, Phys. Rev. Lett. 63, 1245 (1989). 
[12] V. Blackmore, G. Doucas, C. Perry, B. Ottewell, M. F. Kimmitt, M. Woods, S. Molloy, and R. Arnold, First measurements of the longitudinal bunch profile of a $28.5 \mathrm{GeV}$ beam using coherent Smith-Purcell radiation, Phys. Rev. ST Accel. Beams 12, 032803 (2009).

[13] H. L. Andrews, F. B. Taheri, J. Barros, R. Bartolini, V. Bharadwaj, C. Clarke, N. Delerue, G. Doucas, N. Fuster-Martinez, M. Vieille-Grosjean, I. V. Konoplev, M. Labat, S. Le Corre, C. Perry, A. Reichold, and S. Stevenson, Reconstruction of the time profile of $20.35 \mathrm{GeV}$, subpicosecond long electron bunches by means of coherent Smith-Purcell radiation, Phys. Rev. ST Accel. Beams 17, 052802 (2014).

[14] Y. Glinec, J. Faure, A. Norlin, A. Pukhov, and V. Malka, Observation of Fine Structures in Laser-Driven Electron Beams Using Coherent Transition Radiation, Phys. Rev. Lett. 98, 194801 (2007).

[15] P. Karataev, S. Araki, R. Hamatsu, H. Hayano, T. Muto, G. Naumenko, A. Potylitsyn, N. Terunuma, and J. Urakawa, Beam-Size Measurement with Optical Diffraction Radiation at KEK Accelerator Test Facility, Phys. Rev. Lett. 93, 244802 (2004).

[16] J. D. Jackson, in Classical Electrodynamics, 3rd ed. (John Wiley \& Sons, New York, Inc., 1999), pp. 646-654.

[17] G. Kube, H. Backe, H. Euteneuer, A. Grendel, F. Hagenbuck, H. Hartmann, K. H. Kaiser, W. Lauth, H. Schöpe, G. Wagner, T. Walcher, and M. Kretzschmar, Observation of optical Smith-Purcell radiation at an electron beam energy of $855 \mathrm{MeV}$, Phys. Rev. E 65, 056501 (2002).

[18] P. M. van den Berg, Smith-Purcell radiation from a point charge moving parallel to a reflection grating, J. Opt. Soc. Am. 63, 689 (1973).

[19] P. Zhu, Y. Zhu, Y. Hidaka, L. Wu, J. Cao, H. Berger, J. Geck, R. Kraus, S. Pjerov, Y. Shen, R. I. Tobey, J. P. Hill, and X. J. Wang, Femtosecond time-resolved MeV electron diffraction, New J. Phys. 17, 063004 (2015).

[20] R. Li, C. Tang, Y. Du, W. Huang, Q. Du, J. Shi, L. Yan, and $\mathrm{X}$. Wang, Experimental demonstration of high quality $\mathrm{MeV}$ ultrafast electron diffraction, Rev. Sci. Instrum. 80, 083303 (2009).

[21] Y. Muro'Oka, N. Naruse, S. Sakakihara, M. Ishimaru, J. Yang, and K. Tanimura, Transmission-electron diffraction by MeV electron pulses, Appl. Phys. Lett. 98, 251903 (2011).

[22] R. Bartolini, C. Clarke, N. Delerue, G. Doucas, and A. Reichold, Electron bunch profile reconstruction in the few fs regime using coherent Smith-Purcell radiation, J. Instrum. 7, P01009 (2012).

[23] O. Lundh, J. Lim, C. Rechatin, L. Ammoura, A. Ben-Ismail, X. Davoine, G. Gallot, J. P. Goddet, E. Lefebvre, V. Malka, and J. Faure, Few femtosecond, few kiloampere electron bunch produced by a laser-plasma accelerator, Nat. Phys. 7, 219 (2011).

[24] J. M. Krämer, A. Jochmann, M. Budde, M. Bussmann, J. P. Couperus, T.E. Cowan, A. Debus, A. Köhler, M. Kuntzsch, A. L. García, U. Lehnert, P. Michel, R. Pausch, O. Zarini, U. Schramm, and A. Irman, Making spectral shape measurements in inverse Compton scattering a tool for advanced diagnostic applications, Sci. Rep. 8, 1398 (2018).

[25] K. Chouffani, F. Harmon, D. Wells, J. Jones, and G. Lancaster, Determination of electron beam parameters by means of laser-Compton scattering, Phys. Rev. ST Accel. Beams 9, 050701 (2006).

[26] G. Golovin, S. Banerjee, C. Liu, S. Chen, J. Zhang, B. Zhao, P. Zhang, M. Veale, M. Wilson, P. Seller, and D. P. Umstadter, Intrinsic beam emittance of laser- accelerated electrons measured by $x$-ray spectroscopic imaging, Sci. Rep. 6, 24622 (2016).

[27] W. Yan, C. Fruhling, G. Golovin, D. Haden, J. Luo, P. Zhang, and D. P. Umstadter, High-order multiphoton Thomson scattering, Nat. Photonics 11, 514 (2014).

[28] G. Sarri, D. J. Corvan, W. Schumaker, J. M. Cole, A. Di Piazza, H. Ahmed, C. Harvey, C. H. Keitel, K. Krushelnick, S. P. D. Mangles, Z. Najmudin, D. Symes, A. G. R. Thomas, M. Yeung, Z. Zhao, and M. Zepf, Ultrahigh Brilliance Multi-MeV $\gamma$-Ray Beams from Nonlinear Relativistic Thomson Scattering, Phys. Rev. Lett. 113, 224801 (2014).

[29] R. Lai and A. J. Sievers, On using the coherent far IR radiation produced by a charged-particle bunch to determine its shape: I Analysis, Nucl. Instrum. Methods Phys. Res., Sect. A 397, 221 (1997).

[30] H. M. Nussenzveig, Phase problem in coherence theory, J. Math. Phys. (N.Y.) 8, 561 (1967).

[31] J. R. Fienup, Phase retrieval algorithms: A comparison, Appl. Opt. 21, 2758 (1982).

[32] S. I. Bajlekov, M. Heigoldt, A. Popp, J. Wenz, K. Khrennikov, S. Karsch, and S. M. Hooker, Longitudinal electron bunch profile reconstruction by performing phase retrieval on coherent transition radiation spectra, Phys. Rev. ST Accel. Beams 16, 040701 (2013).

[33] W. Press, S. Teukolsky, W. Vetterling, and B. Flannery, Numerical Recipes: The Art of Scientific Computing (Cambridge University Press, Cambridge, England, 1987), pp. 788-812.

[34] E. S. Sarachik and G. T. Schappert, Classical theory of the scattering of intense laser radiation by free electrons, Phys. Rev. D 1, 2738 (1970).

[35] E. Esarey, P. Sprangle, and S. K. Ride, Nonlinear Thomson scattering of intense laser pulses from beams and plasmas, Phys. Rev. E 48, 3003 (1993).

[36] M. Chen, E. Esarey, C. G. R. R. Geddes, C. B. Schroeder, G. R. Plateau, S. S. Bulanov, S. Rykovanov, and W. P. Leemans, Modeling classical and quantum radiation from laser-plasma accelerators, Phys. Rev. ST Accel. Beams 16, 030701 (2013).

[37] I. Ghebregziabher, B. A. Shadwick, and D. P. Umstadter, Stable, tunable, quasimonoenergetic electron beams produced in a laser wakefield near the threshold for selfinjection, Phys. Rev. ST Accel. Beams 16, 031302 (2013).

[38] P. Seller, S. Bell, R. J. Cernik, C. Christodoulou, C. K. Egan, J. A. Gaskin, S. Jacques, S. Pani, B. D. Ramsey, C. Reid, P. J. Sellin, J. W. Scuffham, R. D. Speller, M. D. Wilson, and M.C. Veale, Pixellated $\mathrm{Cd}(\mathrm{Zn}) \mathrm{Te}$ high-energy X-ray instrument, J. Instrum. 6, C12009 (2011). 